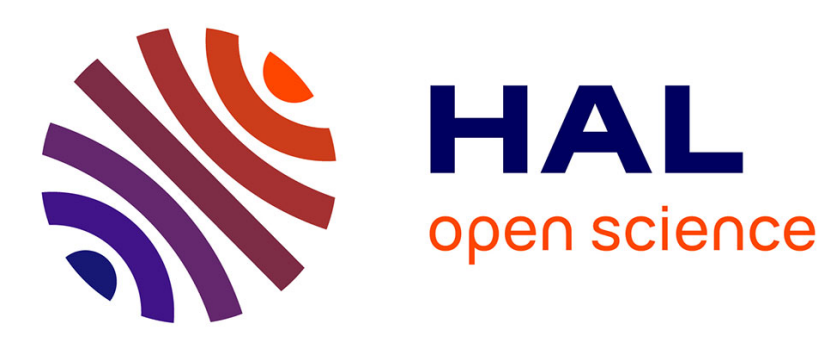

\title{
DNS Study of Roughness-Induced Transition at Mach 6
} Julien Lefieux, Eric Garnier, Neil D. Sandham

\section{To cite this version:}

Julien Lefieux, Eric Garnier, Neil D. Sandham. DNS Study of Roughness-Induced Transition at Mach 6. AIAA AVIATION 2019, Jun 2019, DALLAS, United States. 10.2514/6.2019-3082 . hal-02333650

\section{HAL Id: hal-02333650 https://hal.science/hal-02333650}

Submitted on 25 Oct 2019

HAL is a multi-disciplinary open access archive for the deposit and dissemination of scientific research documents, whether they are published or not. The documents may come from teaching and research institutions in France or abroad, or from public or private research centers.
L'archive ouverte pluridisciplinaire HAL, est destinée au dépôt et à la diffusion de documents scientifiques de niveau recherche, publiés ou non, émanant des établissements d'enseignement et de recherche français ou étrangers, des laboratoires publics ou privés. 


\title{
DNS Study of Roughness-Induced Transition at Mach 6
}

\author{
J. Lefieux* and E. Garnier ${ }^{\dagger}$ \\ DAAA, ONERA, Université Paris Saclay F-92190, Meudon, France \\ N. D. Sandham \\ University of Southampton, Southampton, England SO17 1BJ, United Kingdom
}

In hypersonic flows, it is useful to be able to trip the transition to turbulence upstream of air intakes for example. In the present work, direct numerical simulations have been performed of the flow past an isolated roughness element at Mach 6. First, the capability of two solvers to compute laminar and transitional flow involving freestream disturbances was demonstrated. A series of simulations was then carried out without acoustic perturbation of the freestream. The Reynolds number was increased from 14,000, to 28,000 and then to 40,000. The first two cases remain laminar within the computational domain, whereas the last case undergoes a selfsustained transition to turbulence. A response to a perturbation impulse shows the presence of a varicose mode at the intermediate Reynolds number and a sinuous mode at the largest Reynolds number.

\section{Nomenclature}

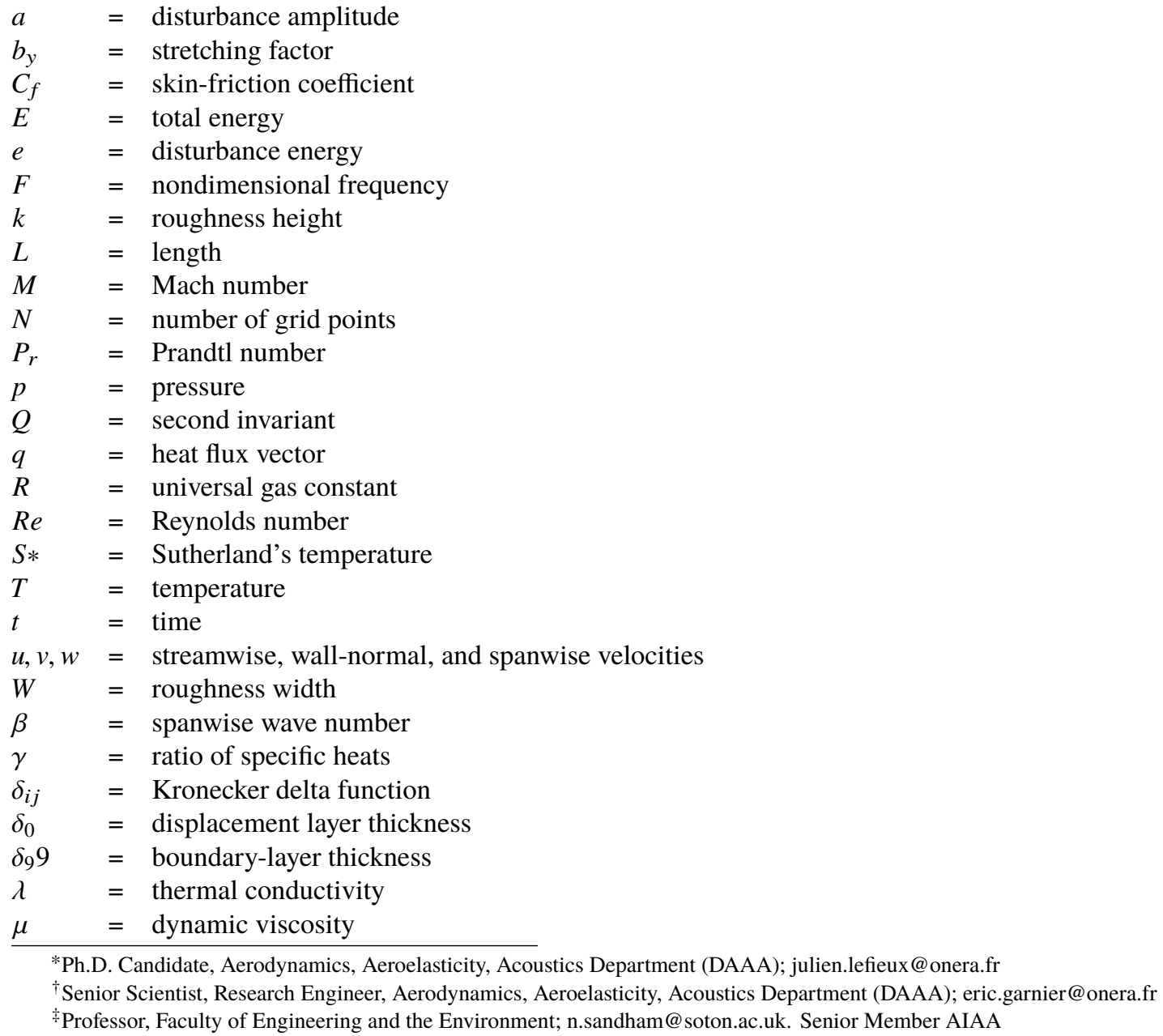




$\begin{array}{ll}\rho & =\text { density } \\ \tau_{i j} & =\text { viscous stress tensor } \\ \phi & =\text { phase } \\ \omega & =\text { circular frequency }\end{array}$

\section{Introduction}

$\mathrm{T}^{\mathrm{N}}$ Mach 6 flows, the weak growth factor of natural instabilities makes it difficult for the flow to go through transition 1 to turbulence. Shock-wave interactions with boundary layers are likely to create separation upstream of engine intakes, thus decreasing the flight performance of future hypersonic vehicles. Prediction of transition in hypersonic flows is also very important because of the impact it has on heat and drag [1]. Roughness elements may be used to act as a source of disturbance for a developing laminar boundary layer to trigger the transition to turbulence upstream of the engine intakes.

An analogy to low-speed flows can be made with respect to the following mechanisms. Due to the recirculation region upstream of the roughness element, a steady horseshoe vortex which is similar to the laminar horseshoe vortex system from Baker [2] is wrapped around the roughness [3][4]. The streamwise vortices and the associated low-momentum streak can lead to a convective Kelvin-Helmholtz instability in the wake, characterized by periodic shedding of hairpin-shaped vortical structures [5], having a dominant role in transition. In hypersonic flow conditions, early experiments focused on the effect of roughness elements on a cone [6]. The importance of using physics based models such as the $e^{N}$ based on linear stability theory over empirical considerations [7] had already been given in [8]. The criteria commonly used to predict the transition, such as the Van Driest and Blumer correlation for cones and hemispheres, $R e_{k}$, or the Shuttle Orbiter correlations, didn't take into account the three-dimensional shape of the roughness. An alternative transition prediction, based on momentum deficit due to the roughness element and taking into account the shape of the roughness was mentioned by Bernardini et al. [9].

Advances in numerical methods and computing capabilities now make it possible to study hypersonic transitional flows with a good accuracy. The factors affecting whether or not a roughness element will be effective in tripping a high-speed flow to turbulence, include the Reynolds number [10], the roughness size, and its shape [11], but also the wall temperature [12] and Mach number [4]. The way in which roughness induces transition is now better understood. As described in [13], a wake with counter-rotating streamwise vortices and a possibly unstable shear layer are generated. The likelihood that transition in the wake of an isolated roughness element will occur depends on "... a race between unsteady fluctuation growth and the rapid relaxation of the basic state toward a spanwise-uniform Blasius flow". The streamwise vorticity behind small roughness elements is likely to grow via instability mechanisms such as stationary crossflow [14], Görtler vortices [15] or transient growth [16]. Transition starting from a receptivity process is also possible, due to an interaction between the roughness and the acoustic waves or other freestream disturbances leading to instability waves.

Global stability analyses have been carried out for subsonic flows but remain relatively scarce for hypersonic flows. Subbareddy et al. [10] performed a dynamic mode decomposition and mentioned a self-sustaining mechanism that causes the flow to transition at Mach 6 and a sufficiently large Reynolds number. Two possible feedback mechanisms were found: one due to the unsteadiness of the strong bow shock inducing pressure variations and providing energy for the periodic vortex motion; another one caused by oscillations in the near wake that trigger motion of the stagnation point which thus affects the bow shock. A global instability whose symmetric core is located in the shear layer separating the outer flow region and the boundary layer immediately downstream of the roughness element was observed at low speeds [17]. A global stability and sensitivity analysis of a three-dimensional Falkner-Skan-Cooke (FSC) boundary layer with a cylindrical surface roughness was performed in [18] where unstable global modes responsible for transition were observed. A global stability analysis made in [19] showed that the global instability of the near-wake region and the associated recirculation region were responsible for the sinuous instability, whereas the whole three-dimensional shear-layer surrounding the central low-speed streak induced the varicose instability.

The aim of the present work is first to validate elsA and OpenSBLI solvers on a laminar and a transitional cases. Then a numerical global stability study based on DNS at Mach 6 will be presented.

\section{Numerical methods}

\section{A. Governing equations}

In this work, the full compressible Navier-Stokes equations are solved for a Newtonian fluid with viscosity $\mu$. 


$$
\begin{gathered}
\frac{\partial \rho}{\partial t}+\frac{\partial \rho u_{j}}{\partial x_{j}}=0 \\
\frac{\partial \rho u_{i}}{\partial t}+\frac{\partial \rho u_{i} u_{j}}{\partial x_{j}}+\frac{\partial p}{\partial x_{i}}=\frac{\partial \tau_{i j}}{\partial x_{j}} \\
\frac{\partial \rho E}{\partial t}+\frac{\partial(\rho E+p) u_{i}}{\partial x_{i}}=-\frac{\partial q_{i}}{\partial x_{i}}+\frac{\partial u_{i} \tau_{i j}}{\partial x_{j}},
\end{gathered}
$$

where $\rho$ is the density, $u_{i}$ is the velocity component in the $\mathrm{i}$-coordinate direction (i=1,2,3); $E=p / \rho /(\gamma-1)+u_{i} u_{i} / 2$ is the total energy per unit mass. The symmetric viscous stress tensor $\tau_{i j}$ is defined as

$$
\tau_{i j}=\frac{\mu}{R e}\left(\frac{\partial u_{j}}{\partial x_{i}}+\frac{\partial u_{i}}{\partial x_{j}}-\frac{2}{3} \frac{\partial u_{k}}{\partial x_{k}} \delta_{i j}\right),
$$

where $\delta_{i j}$ is the Kronecker delta function defined as $\delta_{i j}=1$ for $i=j$ and $\delta_{i j}=0$ for $i \neq j$. The equation of state is considered for the calculations of the fluid properties. Fourier's law of heat conduction is used for the heat flux vector $q_{j}$.

$$
q_{j}=-\frac{\mu}{(\gamma-1) M_{\infty}^{2} \operatorname{PrRe}} \frac{\partial T}{\partial x_{j}} .
$$

The molecular viscosity is computed using Sutherland's law

$$
\mu(T)=T^{\frac{3}{2}} \frac{1+S^{*} / T_{r}^{*}}{T+S^{*} / T_{r}^{*}},
$$

where $S^{*}=110.4 K$ is the Sutherland reference temperature and $T_{r}^{*}=273.15 K$

\section{B. Numerical method}

\begin{tabular}{|c|c|c|c|}
\hline & $\begin{array}{c}\text { elsA } \\
\text { [ONERA] }\end{array}$ & $\begin{array}{c}\text { OpenSBLI } \\
\text { [University of Southampton] }\end{array}$ & $\begin{array}{c}\text { SBLI (Legacy) } \\
\text { [University of Southampton] }\end{array}$ \\
\hline & Finite volume & Finite difference & Finite difference \\
\hline Convective fluxes & AUSM+PW $\left(2^{\text {nd }}\right.$ order) & $5^{\text {th }}$ order WENO & $4^{\text {th }}$ order central difference \\
& Lele $\left(6^{\text {th }}\right.$ order $)$ & $5^{\text {th }}$ order TENO & Total Variation Diminishing (TVD) \\
\hline Viscous fluxes & 5 points centred stencil & $4^{\text {th }}$ order central difference & $4^{\text {th }}$ order central difference \\
\hline Time integration & $2^{\text {nd }}$ order Gear & $3^{\text {rd }}$ order Runge-Kutta & $3^{\text {rd } \text { order Runge-Kutta }}$ \\
& $4^{\text {th }}$ order Runge-Kutta & & \\
\hline
\end{tabular}

Table 1 Numerical parameters for each code used in this study

Table 1 gathers the numerical parameters which have been used in the validation of elsA and OpenSBLI, as well as those used in the reference test case with SBLI (Legacy) [11].

The governing equations are discretized onto a structured grid. OpenSBLI has capability for full three-dimensional curvilinear coordinates. The curvilinear system transforms the equations from a curved to an orthogonal base. A fourth-order central difference method is used to compute the spatial derivatives at the interior points of the domain. The stable boundary treatment of Carpenter et al. [20] is used at the boundaries of the domain to ensure overall fourth-order accuracy. All metric terms for the coordinate transformation are also computed with a fourth order accuracy to avoid additional numerical error. The $3^{\text {rd }}$ order explicit Runge-Kutta low-storage time-stepping scheme described by Jacobs et al. [21] was used in the present simulations. High-order Weighted Essentially non-Oscillatory (WENO) and Targeted Essentially non-Oscillatory (TENO) schemes were used with OpenSBLI in the present work. A validation for the WENO-Z implementation/similarity solution is available in [22]. Instead of selecting only one stencil over the smoothest region of the flow, WENO schemes take a weighted linear combination of all stencils. The weighting is proportional to the local smoothness over each. TENO (Targeted ENO) schemes [23] have lower numerical dissipation than standard 
ENO and WENO methods. Instead of weighting all candidate stencils to build a flux reconstruction, they discard stencils deemed to contain discontinuities while retaining as many smooth stencils as available. In that way it benefits from improved weighting formulations and a staggered stencil arrangement to decrease the number of stencils crossed by a shock.

elsA is based on a finite volume discretization method. It has capability for implicit and explicit time integration schemes. In the first part of the validation (laminar case), the implicit Gear method of $2^{\text {nd }}$ order was used, with 20 Gear sub-iterations in order to increase the time step and reduce the computational time. In the second part of the validation (transitional case), the Runge-Kutta $4^{\text {th }}$ order explicit scheme was used to be compatible with the compact scheme. The second order AUSM+PW [24] scheme was improved from the previous AUSM schemes by introducing pressure-based weight functions. The $6^{\text {th }}$ order compact scheme from Lele [25] was also used in the transitional case, coupled with a $10^{\text {th }}$ order low-pass filter [26].

\section{Global stability method}

The 3D compressible Navier-Stokes equation may be written in a generic way as

$$
\frac{\partial q}{\partial t}=R H S(q)
$$

where $\mathrm{q}$ is the conservative variable vector $[\rho, \rho u, \rho v, \rho w, \rho E]^{T}$. Let us consider $q_{b}$ as a steady flow field obtained with an implicit time-integration scheme, which will be referred to as the base flow. We use this base flow as the initial condition of the simulation. The rate of change of $q$ to satisfy the equilibrium can then be written

$$
\frac{\partial q_{b}}{\partial t}=R H S\left(q_{b}\right)
$$

If the flow is decomposed into its base-flow component $q_{b}$ and a perturbation $q^{\prime}\left(q=q_{b}+q^{\prime}\right)$, then we obtain

$$
\frac{\partial q^{\prime}}{\partial t}=R H S(q)-R H S\left(q_{b}\right)
$$

$R H S\left(q_{b}\right)$ acts like a forcing term in the governing equation. Thus, one can track the time and spatial evolution of an initial disturbance on the base flow, while the flow goes back towards the undisturbed base flow. Therefore, the most unstable (or least damped) mode can be detected. To check the validity of this method, simulations were performed with no initial disturbance and the base flow could be maintained for four flow through times. This method was found to be useful in cancelling out any discretisation differences between the implicit and explicit methods in elsA. Then, white noise disturbances were introduced in the density close to the bump and upstream of it, with a maximum amplitude four orders of magnitude smaller than the freestream.

\section{Comparison of OpenSBLI to elsA on a 3D laminar case}

\section{A. Computational set up}

\section{Computational domain \& roughness element geometry}

The same principle as in [11] was used for the computational domain and for the grid generation. The numerical domain and setup can be seen on figure 1 . The dimensions of the domain $L_{x}, L_{y}$ and $L_{z}$ are respectively 150, 20 and 50 inflow displacement thicknesses. It has periodic boundary conditions at the spanwise boundaries and an isothermal no-slip condition at the wall. OpenSBLI uses Neumann boundary conditions at the outflow and top boundaries. At the inlet, the pressure is extrapolated from the interior of the domain. In elsA, the outflow and top boundaries have a supersonic outlet boundary condition. To make the compact scheme stable, a characteristic boundary condition was used for the outflow, along with a sponge layer. The inflow boundary condition in elsA is decomposed into a subsonic and a supersonic part. The subsonic part is an injection boundary condition where the total pressure and total enthalpy are given in each cell. The supersonic part imposes the conservative variables in each cell, so as to match the full similarity solution.

An isolated roughness element is placed at a distance of $x_{r}=53$ inflow displacement thicknesses from the inlet. The grid was stretched in the wall-normal direction to ensure a sufficient resolution of the boundary layer. The stretching 


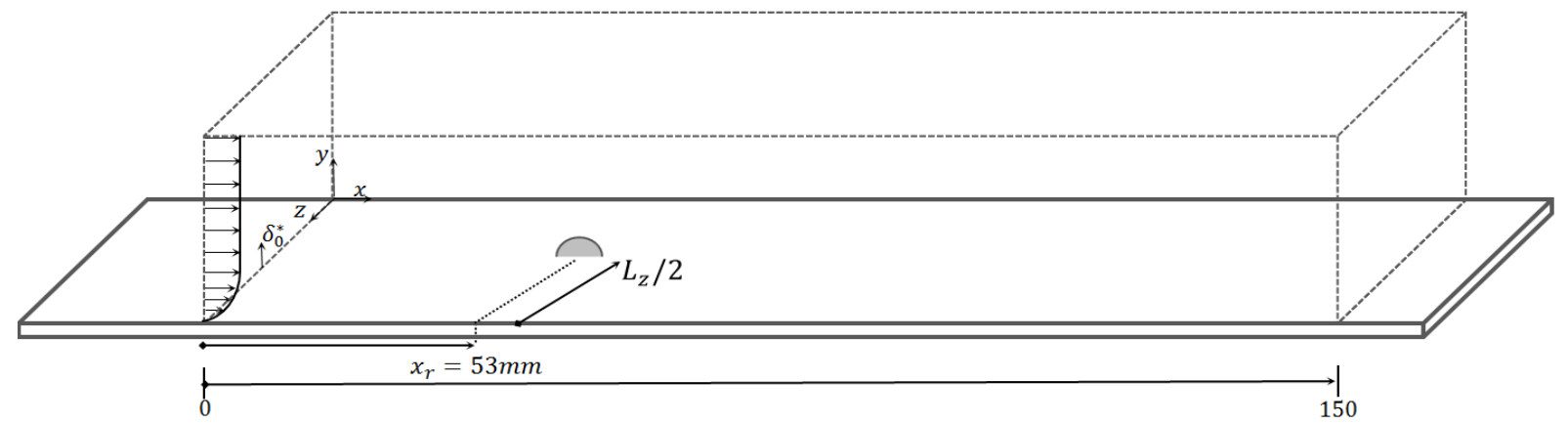

Fig. 1 Computational domain used in the current work

function between the uniform computational grid $(0<\eta<1)$ and the uniform physical grid $\left(y_{0}<y<L_{y}\right)$ is the following:

$$
y=y_{0}+\left(L_{y}-y_{0}\right) \frac{\sinh \left(b_{y} \eta\right)}{\sinh \left(b_{y}\right)},
$$

where $b_{y}=3.19$ is chosen as the stretching factor. The geometry of the bump can be described by the following equation:

$$
y_{0}(\tilde{r})=\frac{-k}{2 \tanh \left(S \frac{W}{k}\right)}\left[\tanh \left(\frac{S}{H}(2 \tilde{r}-W)\right)+\tanh \left(\frac{S}{H}(-2 \tilde{r}-W)\right)\right],
$$

where $\tilde{r}=\sqrt{\tilde{x}^{2}+\tilde{z}^{2}}$ and $S=\cot S^{*}$. The variable $S^{*}$ can be seen as a smoothness factor, which controls the slope of the roughness sides and is equal to $S^{*}=1.35$ for the smooth bump case, and $S^{*}=\frac{\Pi}{4}$ for the flat-top roughness element case.

\section{Polynomial fit for boundary layer}

The initialization of the flow was done based on the full compressible laminar similarity solution in [27]. The laminar boundary-layer profiles for $u, v$ and $T$ were generated numerically in Python during the code generation process in OpenSBLI. The profiles obtained at the inlet were copied over the whole domain for the initialization. This similarity profile is normalized such that the laminar displacement thickness at the domain inflow $\delta_{0}^{*}$ is unity and grows according to

$$
\frac{\delta^{*}\left(\hat{x}^{*}\right)}{\delta_{0}^{*}}=\Delta \frac{\sqrt{2 R e_{\hat{x}^{*}}}}{R e_{0}^{*}}
$$

with

$$
\operatorname{Re}_{\hat{x}^{*}}=\frac{1}{2}\left(\frac{R e_{\delta_{0}^{*}}}{\Delta}\right)^{2}+R e_{\delta_{0}^{*}} \frac{x^{*}}{\delta_{0}^{*}}
$$

where $x^{*}$ is the dimensional streamwise coordinate in a reference frame starting from the leading edge; and $\Delta$ is a scaling factor, depending on Mach number (Mach 6 in the current work) and wall temperature $\left(T_{w}=7.02 T_{r}^{*}\right.$ ) which is equal to $\Delta=9.071$.

\section{B. Grid convergence study}

In the following simulations, a 5th order WENO-Z scheme and the AUSM+PW scheme were used in OpenSBLI and elsA respectively.

A grid convergence study was performed with both elsA and OpenSBLI. Three different grids were used and compared: Fine (900x225x350), Medium (600x150x234), and Coarse (300x100x200). The Fine mesh is close to the one used in [11] which used a grid as fine as in the work of De Tullio and Sandham [28], based on an extensive grid convergence study.

figure 2 shows a pseudo-Schlieren plot of the density gradients to better understand the topology of the flow. Note the edge of the boundary layer relative to the bump height $(\delta / h=1.6)$. One can also notice the separation and reattachment shocks occurring near the bump. The separation/recirculation regions upstream and downstream of the bump are shown in red, using contours of small negative streamwise velocity $\left(u=-10^{-5}\right)$. The recirculation regions are better seen 


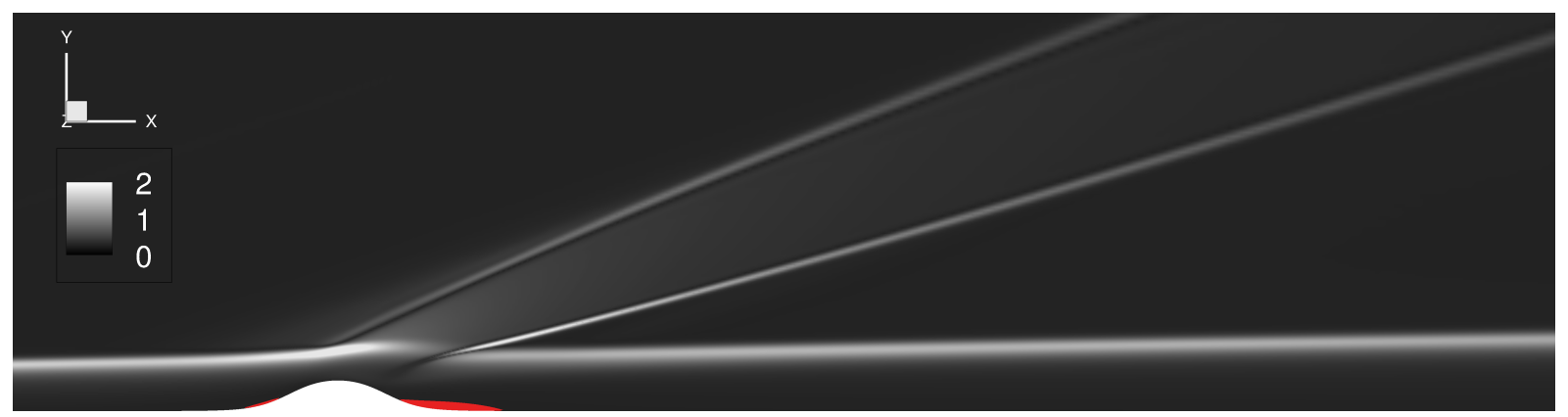

Fig. 2 Pseudo-Schlieren plot showing density gradients in the roughness centreplane. Red region showing the recirculation regions by using contours of small negative streamwise velocity $\left(u=-10^{-5}\right)$

on figure 3. The convergence study is consistent when looking at the recirculation region boundaries. The Medium and Fine grids give the same results whereas the Coarse mesh under-predicts the change in $v$ or $\rho$ around the bump, in regions of high shear-stress.

\section{Results of the comparison}

For elsA, the results obtained with AUSM+PW are presented here. Jameson and Roe schemes were also used and gave similar results. The Fine mesh was used for the comparison of elsA and OpenSBLI. Very good agreement was reached between them. As can be seen on figure 4 the change in velocity in all three directions is predicted the same in both codes. The only noticeable discrepancies can be found on $v$ for instance at $x=53$ or $x=58$. The maximum deviation found at the reversed flow peak location (most negative value of $v$ ) at $x=53$ by elsA with respect to OpenSBLI is $2.5 \%$. $\rho$ and $T$ profiles also show good agreement between the two codes.

This first comparison validates the computation of viscous and metric terms in OpenSBLI, as well as the ability of $2^{\text {nd }}$ order schemes in elsA to predict hypersonic laminar flows accurately.

\section{Comparison of elsA and OpenSBLI to SBLI on a transitional case}

\section{A. Computational set-up}

Validation of elsA and OpenSBLI with reference to the work done in [11] with the code SBLI was carried out. The comparison is made on a transitional case, which is more discriminating for the choice of the numerical schemes used for the simulations and more sensitive to boundary conditions. The same grid as in [11] was used for this case. The numerical domain and setup can be seen on figure 1 . The grid and domain sizes are shown in Table 2 . The domain was shortened in the streamwise direction from 300 to 190 inflow displacement thicknesses as the transition, which we are interested in, is supposed to occur before the end of the domain.

\begin{tabular}{|c|c|c|}
\hline & SBLI & OpenSBLI \\
\hline Lx x Ly x Lz & $300 \times 20 \times 50$ & $190 \times 20 \times 50$ \\
\hline$N_{x} \times N_{y} \times N_{z}$ & $2415 \times 205 \times 468$ & $1600 \times 205 \times 468$ \\
\hline$\Delta x$ & {$[0.15$ (upstream), 0.05 (roughness), 0.135 (downstream)] } \\
\hline$\Delta z$ & \multicolumn{2}{|c|}{$[0.04$ (roughness), 0.15] } \\
\hline
\end{tabular}

Table 2 Grid and domain sizes used for the transitional case. Values of $\Delta x$ are the grid spacing upstream, near and downstream of the roughness (Lengths are in $\mathbf{m m}$ )

Once again the initialization of the flow was done based on the full compressible laminar similarity solution. The Reynolds number based on the inflow displacement thickness is now $\operatorname{Re}_{\delta_{0}^{*}}=14,000$. The freestream reference temperature is $T_{r}^{*}=273.15 \mathrm{~K}$, and the static pressure is $p=9451.319 \mathrm{~Pa}$. A normalization of the parameters was performed on $\rho, u$ and $T$, leaving an adimensionalized static pressure of $p_{a d}=0.01984$. Figure 5 shows what the 


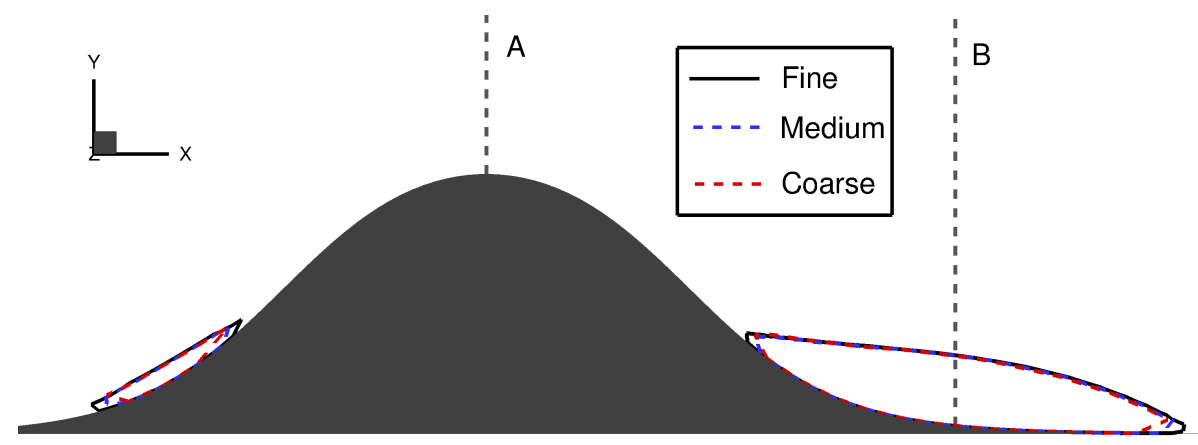

(a) Recirculation regions
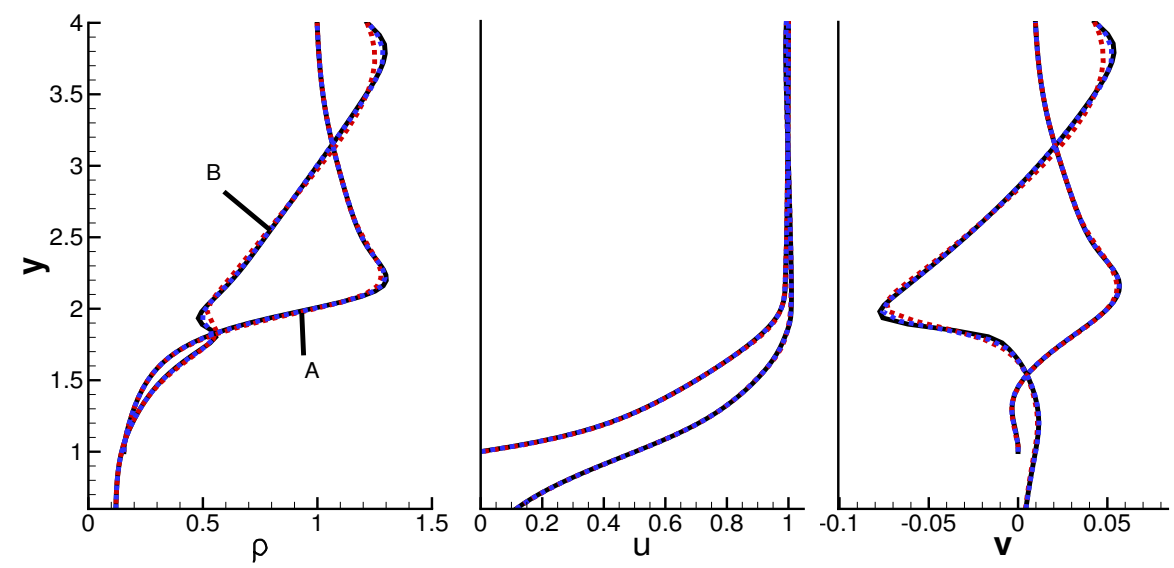

(b) Boundary-layer profiles at $x=53$ (A) and $x=60$ (B)

Fig. 3 OpenSBLI grid convergence study in the symmetry plane
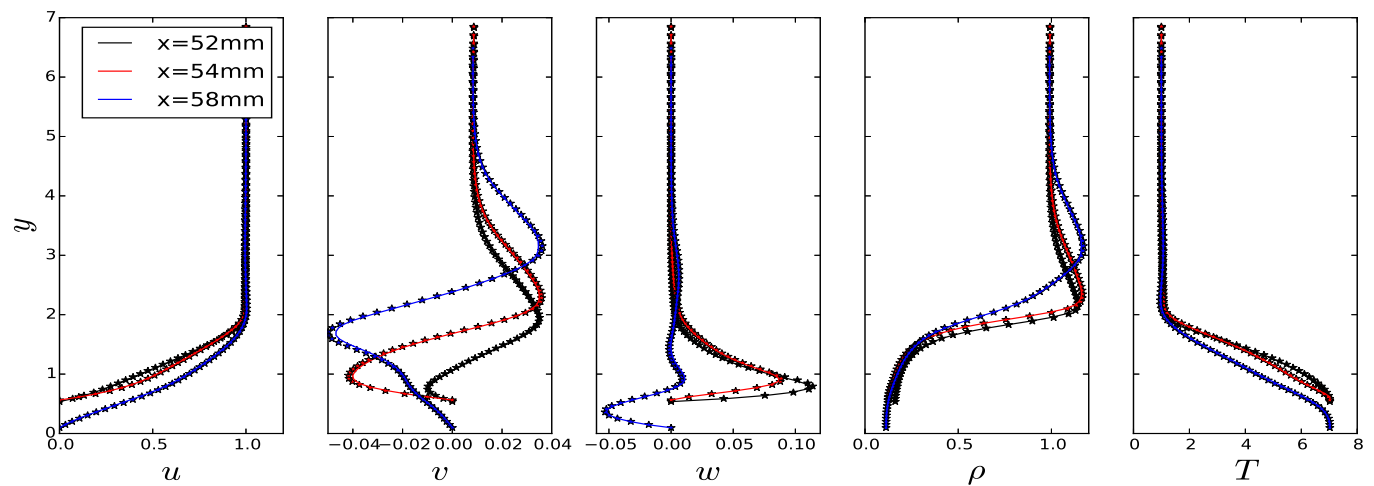

Fig. 4 elsA (lines) / OpenSBLI (symbols) comparison with the Fine mesh - Flow profiles at $\mathrm{z}=2.8$ 


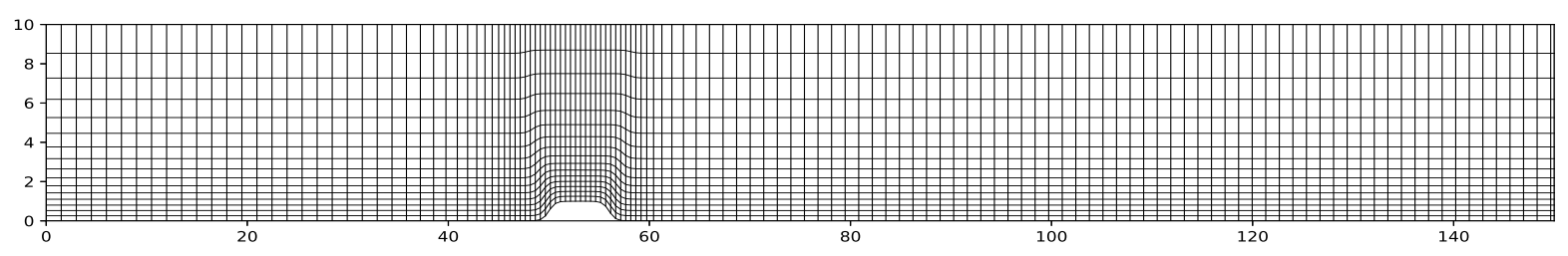

Fig. 5 Computational grid in the roughness centreplane plane (shown every $10^{\text {th }}$ grid lines)

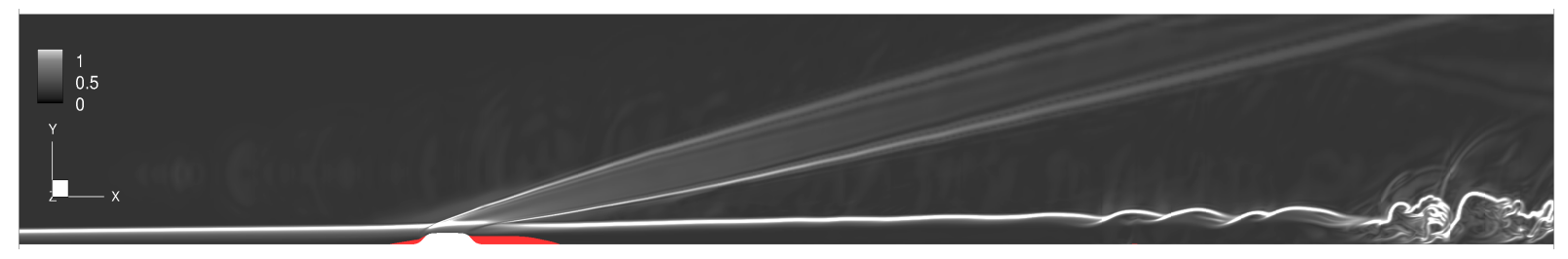

Fig. 6 Instantaneous contours of density gradient magnitude in the centreplane showing transitional flow at the end of the domain, computed with elsA. Red zones showing the recirculation regions.

computational grid looks like. It is plotted every $10^{\text {th }}$ grid line for better clarity.

In order to keep the Reynolds number moderate and still be able to trigger instabilities, acoustic disturbances were introduced in the freestream in the work of [11], with a 3-dimensional source placed at $x_{f}=12.0$ and $y_{f}=6.0$. The same acoustic forcing was used in the simulations with elsA and OpenSBLI solvers. This forcing consists of a source term $s$, added to the right hand side of the continuity equation and described by

$$
s(x, t)=a \exp \left(-r^{2}\right) \sum_{m=0}^{M} \sum_{n=1}^{N} \cos \left(\beta_{m} z+\phi_{m}\right) \sin \left(\omega_{n} t+\phi_{n}\right),
$$

where $a=3 \times 10^{-4}$ is the amplitude, $r=\sqrt{\left(x-x_{f}\right)^{2}+\left(y-y_{f}\right)^{2}}$ gives the distribution, $\beta_{m}=2 \pi m / L_{z}$ is the spanwise wave number and $\omega_{n}=2 \pi n F_{0}$ is the frequency with $F_{0}=0.02$. The acoustic source has thus $M=20$ spanwise modes and $N=20$ temporal modes to mimic the broadband nature of acoustic fluctuations in turbulent boundary layers. The simulation integration time of statistical quantities is governed by the lower and upper bound of the frequency, while the spanwise wave number range sets the required grid resolution. $\phi_{n}$ and $\phi_{m}$ are random phases which prevent large spikes in the imposed forcing. These random phases used in the older simulations with SBLI were unknown meaning that different phases with elsA and OpenSBLI are likely to be present.

\section{B. Results}

Figure 6 shows the instantaneous contours of density gradient magnitude in the centreplane, computed with elsA. The contour range was adjusted to highlight the interaction between the density fluctuations caused by the acoustic forcing, the roughness element and the boundary layer, leading to transition at the end of the domain. Again the red zones show the recirculation regions which are larger than in the laminar case, due to the shape of the roughness element which is now a flat-top cylindrical roughness element. First, elsA and OpenSBLI can be compared qualitatively, by looking at figure 7 which shows instantaneous $Q$ criterion iso-surfaces $(Q=0.003)$ coloured by the streamwise vorticity at different time instances. Good agreement is obtained in terms of the flow structures predicted by both solvers. They display longitudinal streaks, rolling up in the wake of the roughness element, forming hairpin-like vortices that eventually break down to turbulence.

A quantitative comparison of the three solvers is made by looking at the skin friction coefficient, averaged over one period of the forcing along the domain centreline, as shown on figure 8(a) Good agreement is obtained in the laminar part of the flow and the recirculation regions are predicted the same by both codes. The disturbance energy $e$ is integrated over $99 \%$ of the boundary-layer thickness at the roughness centreline and shown for each code as a function of $x$ in figure 8(b). Also the average exponential growth rate $\sigma$ is computed as the average growth rate $\sigma$ in the range of $100<\mathrm{x}<140$, and shown in the legend of figure $8(\mathrm{~b})$. The receptivity process is linear, and the boundary-layer disturbances grow exponentially before reaching saturation, indicating the occurrence of nonlinear interactions. The 


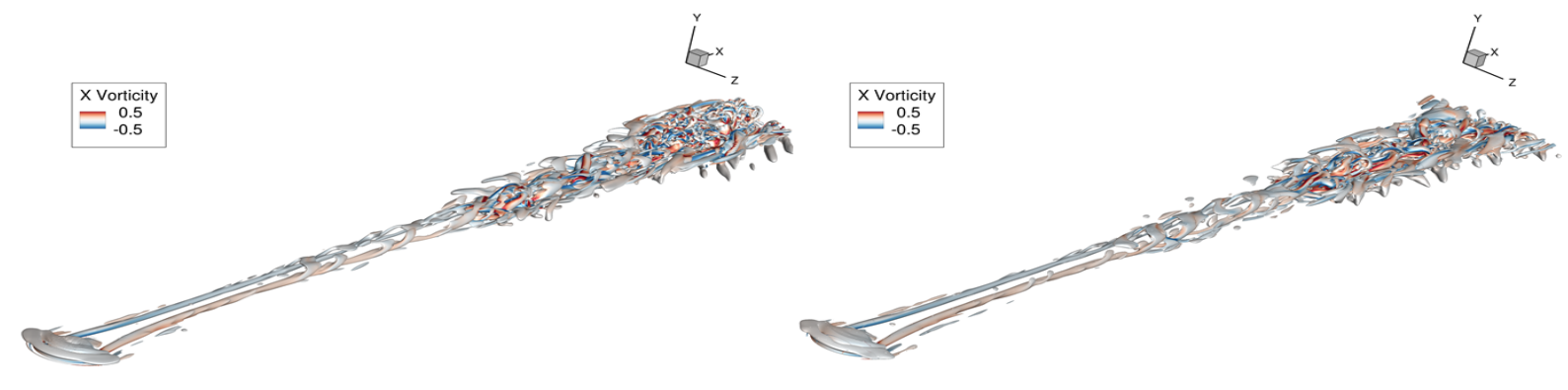

Fig. 7 Instantaneous $Q$ criterion iso-surfaces $(Q=0.003)$ for OpenSBLI (left) and elsA (right), coloured by the streamwise vorticity

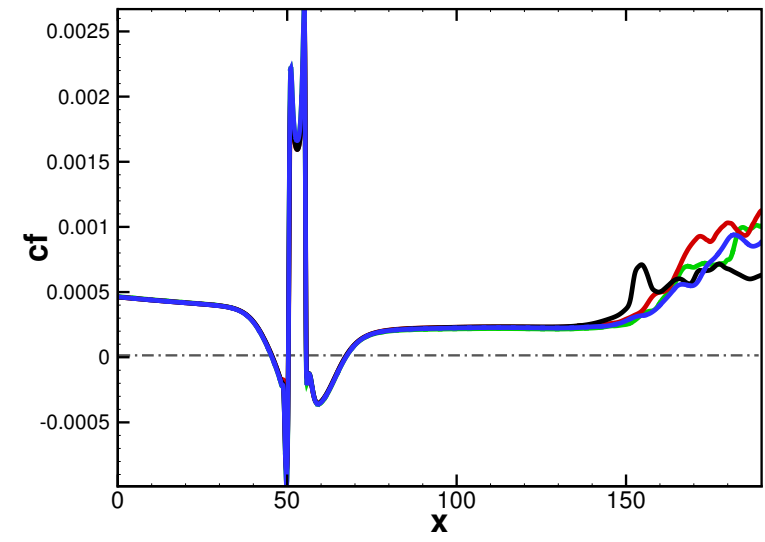

(a) Skin friction coefficient

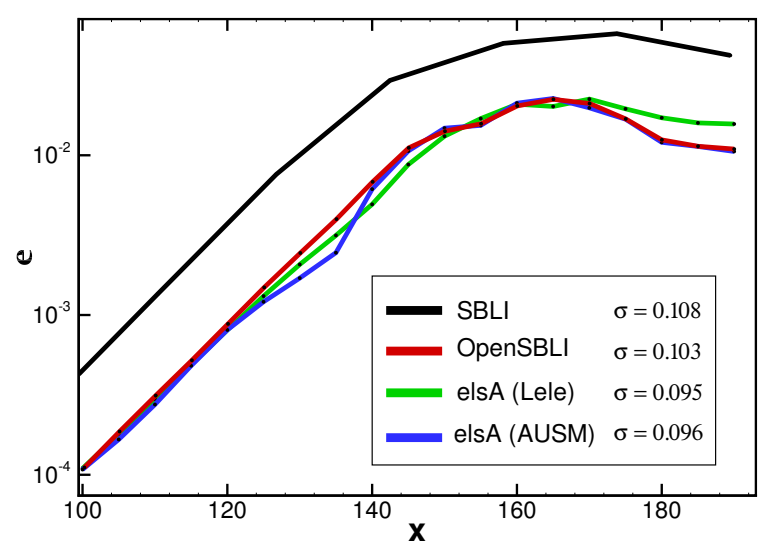

(b) Integrated disturbance energy

Fig. 8 Comparison of elsA, OpenSBLI and SBLI in the symmetry plane.

black solid line in figure 8(b) corresponding to SBLI results [11] exhibits an offset due to different forcing. The work of [11] showed minor impact of the freestream disturbance amplitude on the growth rate of linear instabilities. This is also observed in the present validation, where the exponential growth rates of all four cases agree very well. The breakdown to turbulence, however, takes place earlier for the SBLI test case due to the increased integrated forcing energy compared to the computations by elsA and OpenSBLI.

\section{Global stability study}

\section{A. Numerical methodology}

\section{Numerical domain and grid}

The same roughness element as in section $\mathrm{V}$ was used for this study. The domain was shortened in the streamwise direction to $L_{x}=90$ and in the spanwise direction to $L_{z}=40$. The roughness element is now placed at a distance of 40 inlet displacement thicknesses from the inflow. The Reynolds number was increased from 14,000 to 28,000 and then to 40,000 .

A grid convergence study was made at $R e=40,000$ with OpenSBLI, with a Coarse, Medium and Fine grids having respectively $\left(N_{x}, N_{y}, N_{z}\right)=(800,100,200),(1200,150,300)$ and $(1800,225,450)$ grid points in each direction. This showed consistency of the results, in terms of whether or not transition occurs, although refining the grid resulted in earlier transition, as shown by the increase of skin-friction coefficient on figure 9, suggesting that even finer grids are needed. Under these considerations, the number of grid points in each direction was chosen as $\left(N_{x}, N_{y}, N_{z}\right)=(1800,300,875)$ 


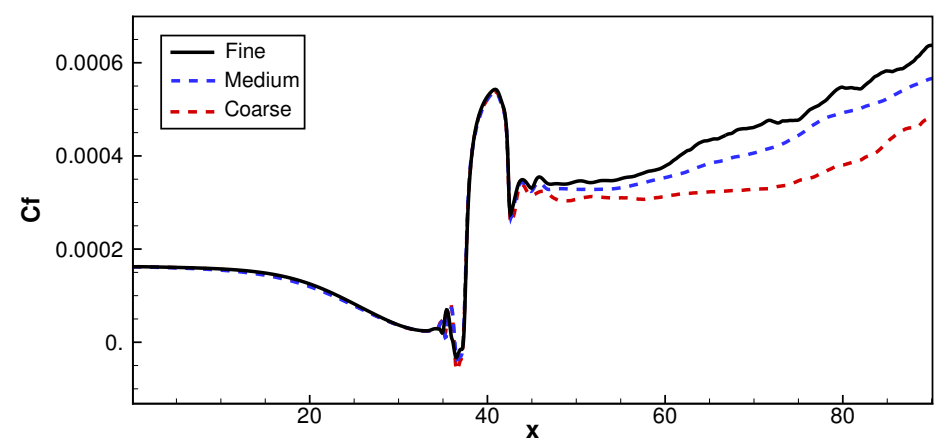

Fig. 9 Grid convergence study for the $R e=40,000$ case - Skin friction coefficient averaged over span and time

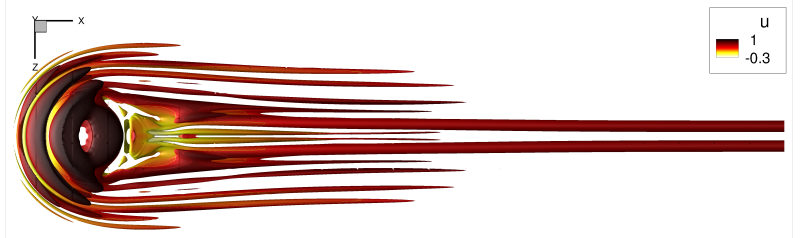

(a) $\mathrm{Re}=28,000$

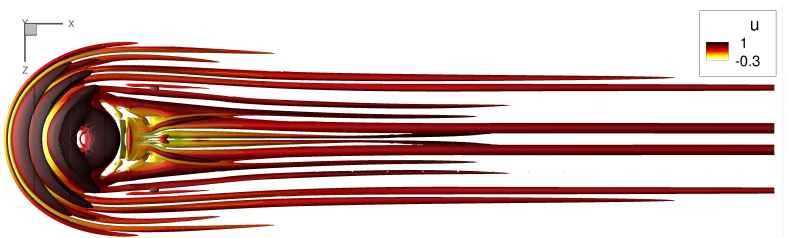

(b) $\mathrm{Re}=40,000$

Fig. 10 Isosurface of $\mathbf{Q}$ criterion $Q=0.005$ of the base flows, coloured by the streamwise velocity

resulting in 472.5 million grid points. This led to $\Delta x^{+}<6.8, \Delta z^{+}<5.7$ and $\Delta y^{+}<1.2$ for the first cell above the wall.

\section{Calculation of the laminar base flow}

The numerical solution of the laminar base flow was obtained by using elsA. The convective fluxes were computed using a second order Roe scheme, along with a minmod limiter. Time integration was achieved using an implicit (backward Euler) scheme. Once again the flow is initialized with the compressible similarity solution for the boundary layer. The system is integrated in time until a decrease of sixth order of magnitude in the average residual is achieved. This method was found to be successful in obtaining a steady base flow, even though the $R e=40,000$ undergoes a self-sustained transition to turbulence. Figure 10 shows iso-surfaces of $Q$ criterion $(Q=0.005)$ coloured by the streamwise velocity for both base flows.

\section{B. Results}

\section{Reynolds number comparison}

Unsteady simulations were first carried out with OpenSBLI, using a $5^{\text {th }}$ order TENO scheme for the spatial discretization. Figure 11(a) compares the three Reynolds number in terms of vortical structures induced by the roughness. It is clear that case $R e=14,000$ (i) stays laminar, whereas case $R e=28,000$ (ii) is on the edge to transition. Case $R e=40,000$ (iii), however, is experiencing a self-sustained transition to turbulence. As can be seen in figure 11(b) which is based on a time-averaged flow field, increasing the Reynolds leads to a larger upstream recirculation region (shown by the red line) but a smaller downstream recirculation region in the symmetry plane. The highest Reynolds number cases also exhibits more vortices within the horseshoe vortex system. The time-averaged pressure contours are also shown on figure 11(b)

The $R e=40,000$ case undergoes a self-sustained transition to turbulence, in the sense that no artificial disturbances are needed for instabilities to develop and to sustain by themselves. This is likely to be due to the presence of a global instability with its origin near the roughness element. The fact that the bow shock wrapping around the roughness-element is a potential source of vortex structure unsteadiness was mentioned in [10] and cannot be ruled out. Figure 12 shows an instantaneous of the Q criterion coloured by streamwise vorticity. One can notice the presence of 

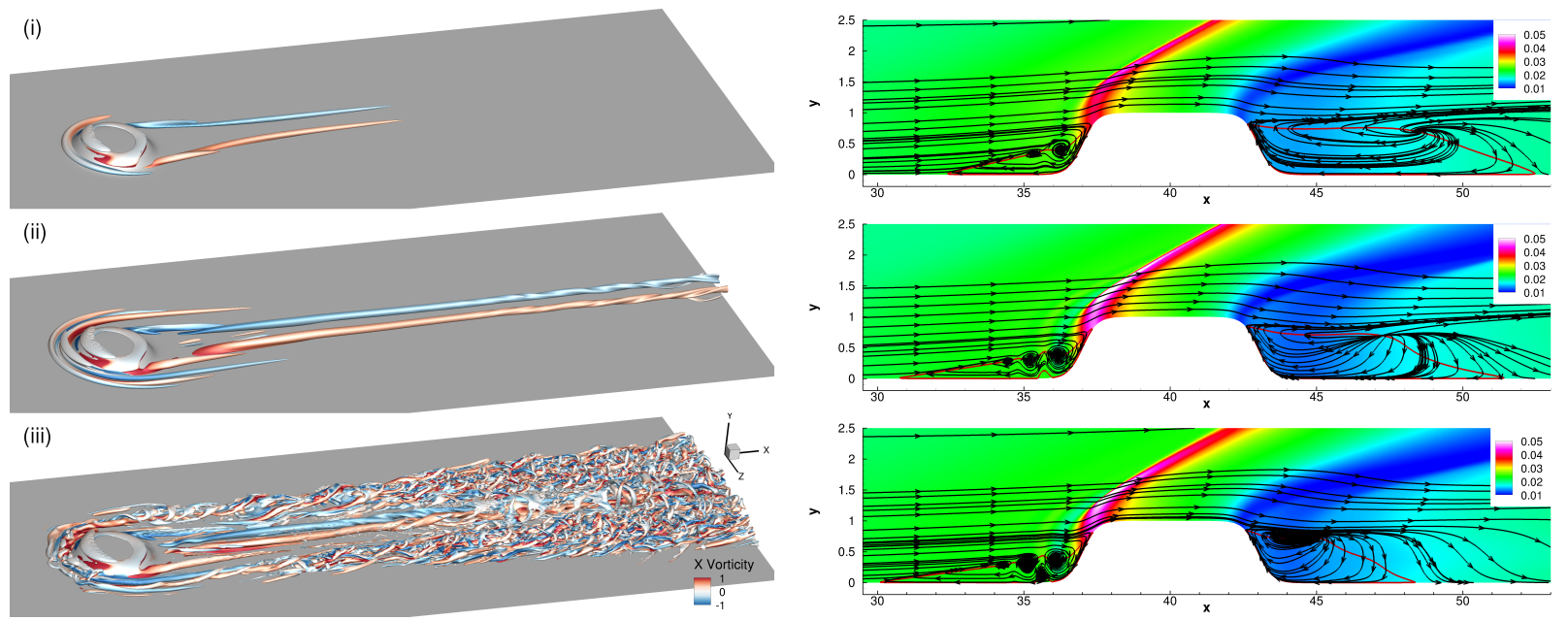

(a) Isosurface of $\mathrm{Q}$ criterion $Q=0.05$

(b) Time-average pressure in the symmetry plane. The red line shows separated regions.

Fig. 11 Reynolds number comparison. From top to bottom, $R e=14,000$ (i), $R e=28,000$ (ii) and $R e=40,000$ (iii)

counter-rotating streamwise vortices away from the symmetry plane due to the upstream separation-induced vortices wrapping around the roughness. As described in [29], the shear layers bounding these streaks roll up and result in hairpin-shaped structures. Close to the symmetry plane and downstream of the roughness, large streamwise streaks are also present, inducing the formation of hairpin-shaped structures. Farther downstream, these trains of hairpin vortices eventually lead to the formation of secondary vortices, resulting in the spanwise spreading of turbulent structures, in a similar way to the results of [30].

\section{Response to a perturbation impulse at $R e=28,000$ and $R e=40,000$}

The response to a perturbation impulse was carried out at $R e=28,000$ and $R e=40,000$, following the method described in section III.C. The initial disturbance consists of an increment of $1 \times 10^{-4}$ in the density, in a region of $3 \times 3$ grid points around the location $(x, y, z)=(0.33,0.5,0.75)$, corresponding to a location within the boundary layer, upstream of the roughness element. This effectively introduces a disturbance with a sharp-edged distribution, which will excite a range of frequencies at low amplitude. The simulation restarts from the base flow with this initial disturbance and the response of the flow to this perturbation is monitored for four flow-through domains. First of all, the power spectral density (PSD) computed from the wall pressure perturbation response at $(x, z)=(88,0.75)$ is shown on figure 13. The PSD was adimentionalized by the variance of the signal for better comparison. The non-dimensional frequencies $F=0.14$ and $F=0.24$ are the most amplified frequencies for $R e=28,000$ and $R e=40,000$ respectively. $F=0.14$ was often mentioned in the literature [28] and was related to a varicose instability (or Mack mode). $F=0.24$ is also mentioned by [31] as being related to a sinuous mode.

Figure 14 shows the time histories of the wall pressure perturbation $p$ for different streamwise location. On the vertical axis, $x$ indicates the streamwise distance. The maximum amplitude of the pressure perturbation obtained at the end of the domain $(x=90)$ is $\pm 2.5 \times 10^{-6}$ and $\pm 5 \times 10^{-8}$ for $R e=28,000$ and $R e=40,000$ respectively. In the $R e=28,000$ case (figure 14(a) , a first wave-packet induced by the initial disturbance can be observed $(0<t<100)$. It convects downstream towards the end of the domain. At $t=150$, a second wavepacket has reached the end of the domain and undergoes an exponential growth, before converging towards a self-sustained oscillatory state (red line in figure 14(a) . In the $R e=40,000$ case (figure 14(b) , the initial disturbance once again convects downstream, but the exponential growth at $x=90$ occurs more rapidly. Even in the absence of disturbances, oscillations self-sustain in the wake of the roughness element, indicating the presence of a global instability. Figure 15 shows the remaining modes at $t=180$ by displaying the density perturbation. The structures left at this time show a varicose (symmetrical) mode at $R e=28,000$, whereas a sinuous instability is present for the highest Reynolds number. The shape of the structures is qualitatively similar to the modes found by De Tullio [31] at a lower supersonic Mach number $(\mathrm{M}=2.5)$. 


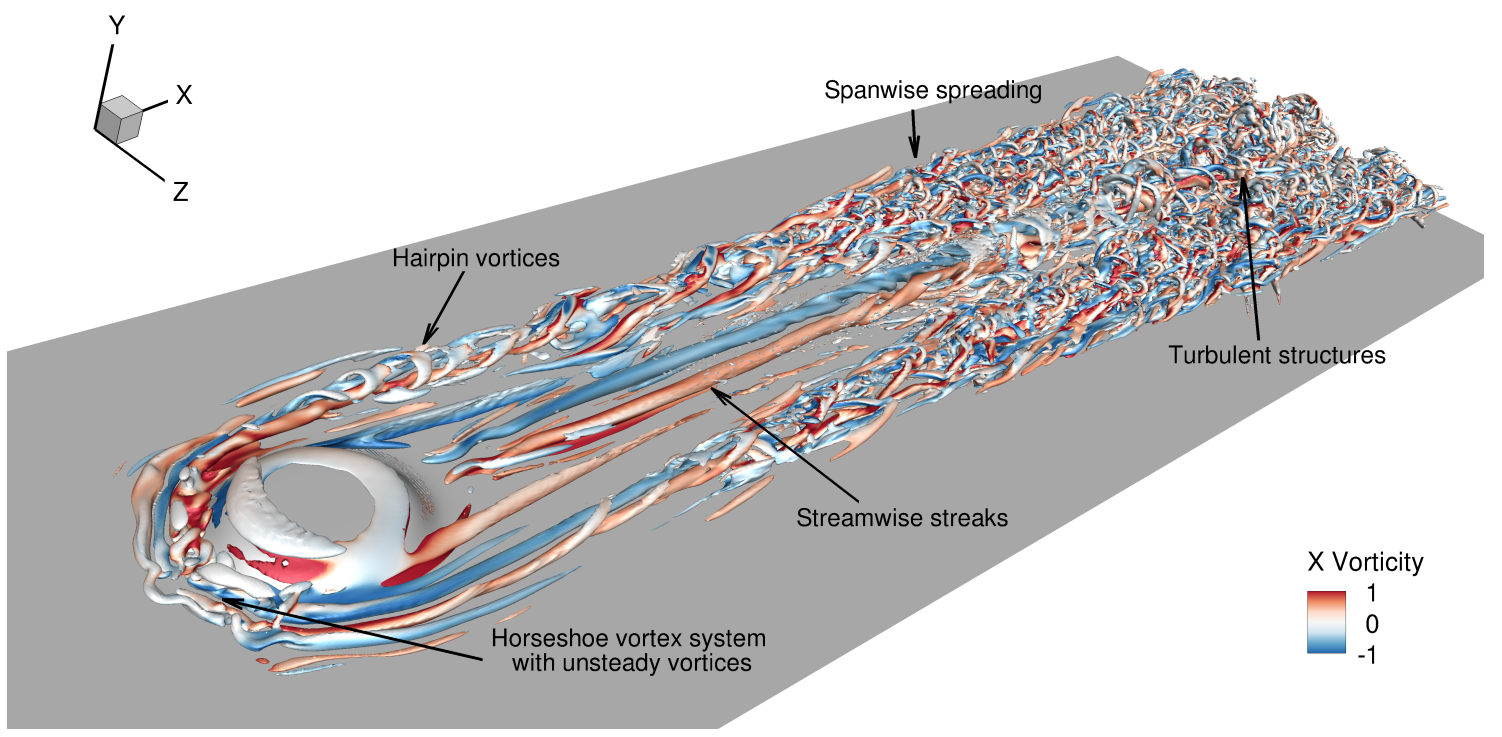

Fig. 12 Visualization of instantaneous $\mathbf{Q}$ criterion coloured with streamwise vorticity $w_{x}$ at $R e=40,000$

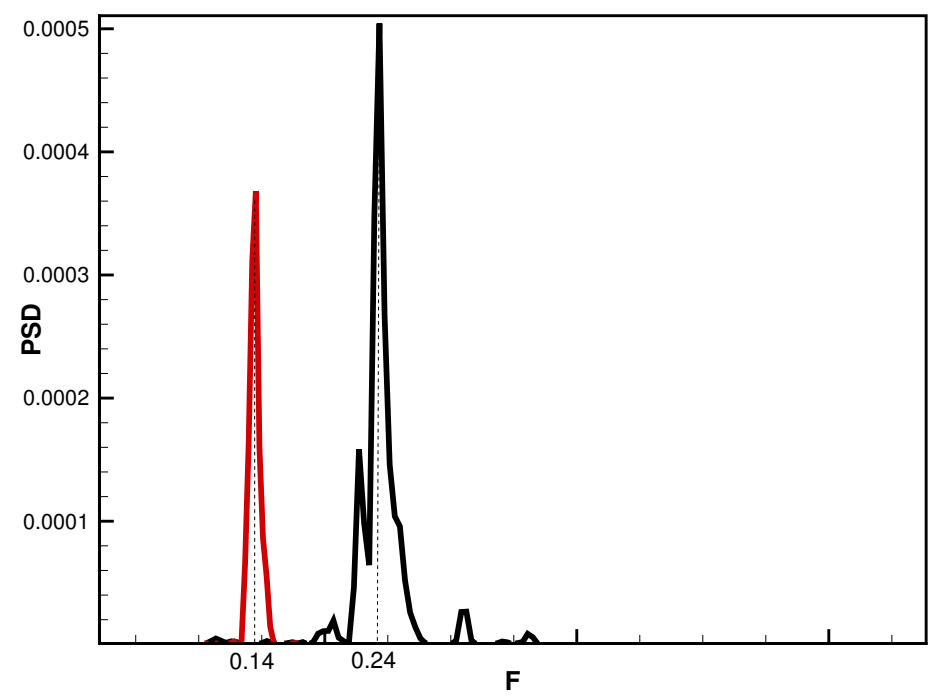

Fig. 13 Power Spectral Density (PSD) computed from the wall pressure perturbation response at $(x, z)=$ $(88,0.75)$, normalized by the variance of the signal for each Reynolds number 


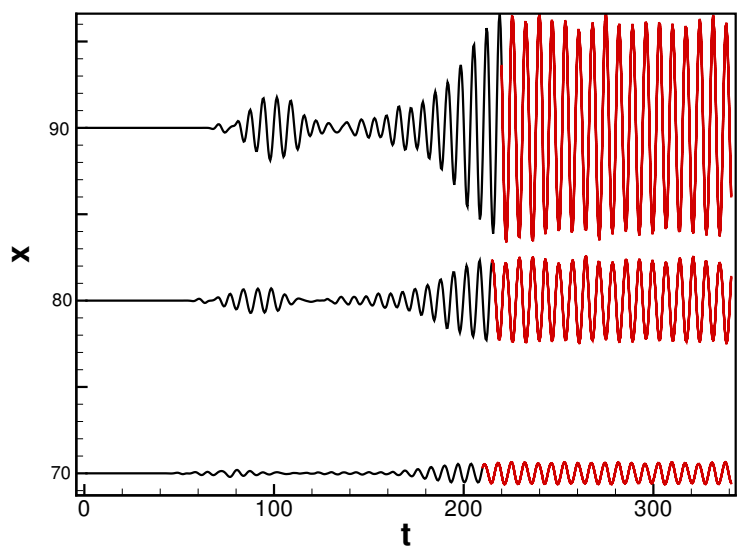

(a) $R e=28,000$

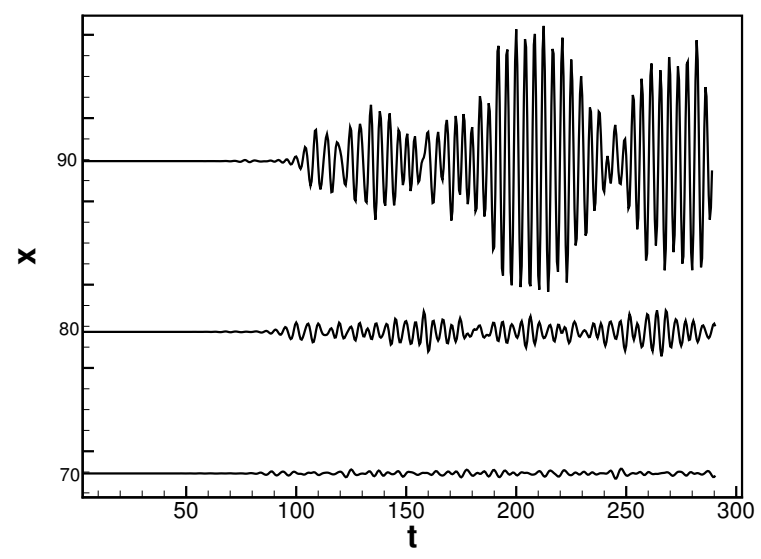

(b) $R e=40,000$

Fig. 14 Time histories of wall pressure perturbation $p$, with streamwise distance indicated on the vertical axis, in the $x-y$ plane at $z=0.75$. The red part shows saturation where the regime is no longer linear.

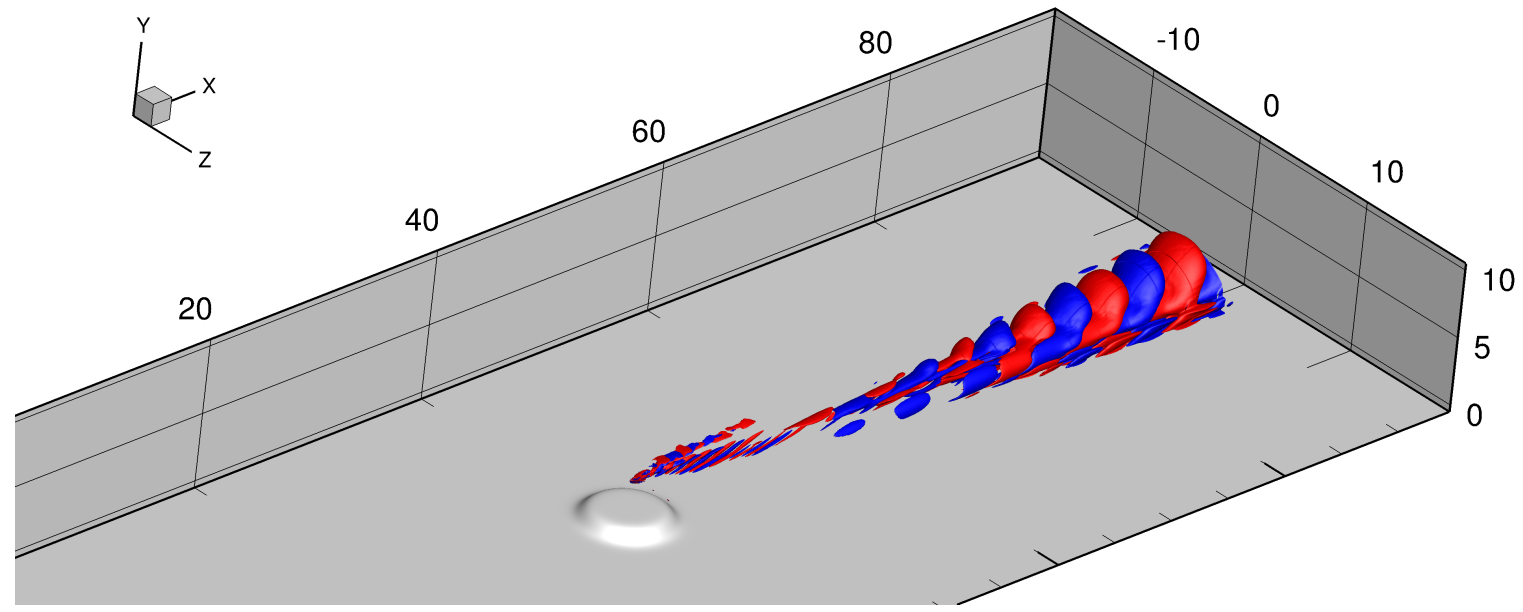

(a) $R e=28,000$, varicose mode $F=0.14, \rho^{\prime}= \pm 5 \times 10^{-7}$

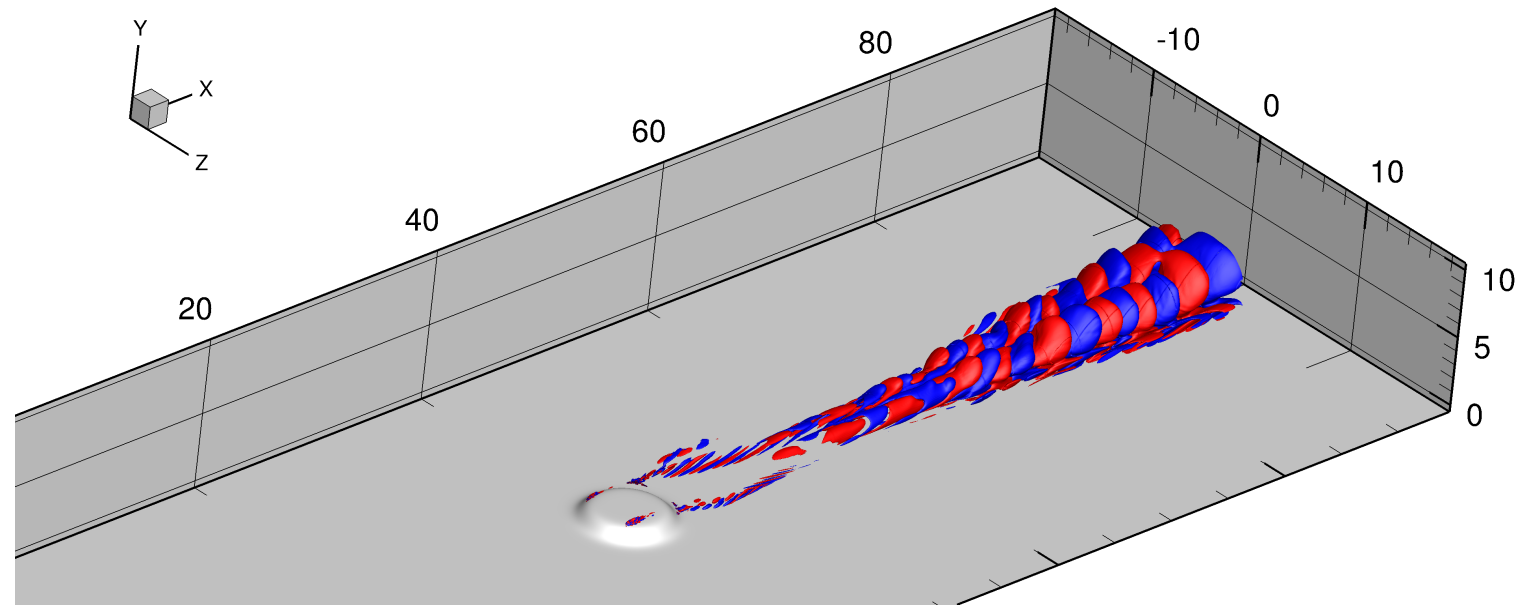

(b) $R e=40,000$, sinuous mode $F=0.24, \rho^{\prime}= \pm 1 \times 10^{-6}$

Fig. 15 Isosurfaces of the density perturbations showing the remaining most unstable modes at $t=180$ 


\section{Conclusion}

The flow over an isolated roughness element has been studied using direct numerical simulations at Mach 6 . Comparison of elsA and OpenSBLI was made on a laminar case $(R e=8,200)$ over a smooth bump and was found to be successful. This validates the computation of metric and viscous terms in OpenSBLI as well as the ability of $2^{\text {nd }}$ order schemes in elsA to predict hypersonic laminar flows accurately. Validation of elsA and OpenSBLI with respect to SBLI on a transitional case $(R e=14,000)$ where the transition is triggered by acoustic perturbations was also satisfying. The second order AUSM+PW scheme as well as the $6^{\text {th }}$ order compact scheme in elsA are found to give close results to the $5^{\text {th }}$ order TENO scheme in OpenSBLI. A global stability study has been carried out using a DNS approach. The flow for three Reynolds numbers was compared without addition of acoustic disturbances. The lowest Reynolds number case stays laminar. At the intermediate Reynolds number, the flow in on the edge to transition but remains laminar within the simulated domain. The largest Reynolds number undergoes a self-sustained transition to turbulence. This is due to the presence of a global instability. The response to an initial perturbation impulse at both the intermediate and large Reynolds numbers shows evidence of a varicose instability (at $F=0.14$ ) and a sinuous instability (at $F=0.24$ ) respectively. The origin of the self-sustained oscillatory mechanism has not yet been determined although the instability appears to be linked to the downstream recirculation region and to the shear-layer induced by the roughness element.

\section{Acknowledgments}

This work was funded by the "Ministère des armées - Direction Générale de l'Armement (DGA/DSTL)" as part of the UK-FR PhD program. It was performed using resources provided by the Cambridge Service for Data Driven Discovery (CSD3) operated by the University of Cambridge Research Computing Service (http://www.csd3.cam.ac.uk/), provided by Dell EMC and Intel using Tier-2 funding from the Engineering and Physical Sciences Research Council.

\section{References}

[1] Wang, X., Zhong, X., and Ma, Y., "Response of a hypersonic boundary layer to wall blowing-suction," AIAA Journal, Vol. 49, No. 7, 2011, pp. 1336-1353.

[2] Baker, C. J., “The laminar horseshoe vortex," Journal of Fluid Mechanics, Vol. 95, No. 2, 1979, p. 347-367. doi: $10.1017 /$ S0022112079001506.

[3] Bartkowicz, M., Subbareddy, P., and Candler, G., "Numerical Simulations of Roughness Induced Instability in the Purdue Mach 6 Wind Tunnel," 40th Fluid Dynamics Conference and Exhibit, 2010.

[4] Iyer, P. S., Muppidi, S., and Mahesh, K., "Roughness-induced transition in high speed flows," 49th AIAA Aerospace Sciences Meeting including the New Horizons Forum and Aerospace Exposition, 2011. doi:10.2514/6.2011-566.

[5] Acarlar, M. S., and Smith, C. R., "A study of hairpin vortices in a laminar boundary layer. Part 1. Hairpin vortices generated by a hemisphere protuberance," Journal of Fluid Mechanics, Vol. 175, 1987, p. 1-41. doi:10.1017/S0022112087000272.

[6] Van Driest, E. R., and Blumer, C. B., "Boundary layer transition at supersonic speeds - Three dimensional rougness effects (spheres)," Journal of Aerosol Science, Vol. 29, 1962, p. 54.

[7] Reshotko, E., “Is $R e_{\theta} / M_{e}$ a meaningful transition criterion?” AIAA Journal, Vol. 45, No. 7, 2007, pp. 1441-1443.

[8] Morkovin, M. V., Reshotko, E., and Herbert, T., "Transiton in open flow systems: a reassessment," Bull. Am. Ohys. Soc., 1994.

[9] Bernardini, M., Pirozzoli, S., and Orlandi, P., "Parameterization of Boundary-Layer Transition Induced by Isolated Roughness Elements,” AIAA, Vol. 52, No. 10, 2014, pp. 2261-2269. doi:10.2514/1.J052842.

[10] Subbareddy, P. K., Bartkowicz, M. D., and Candler, G. V., "Direct numerical simulation of high-speed transition due to an isolated roughness element,” Journal of Fluid Mechanics, Vol. 748, 2014, pp. 848-878. doi:10.1017/jfm.2014.204.

[11] Van den Eynde, J. P., and Sandham, N. D., "Numerical Simulations of Transition due to Isolated Roughness Elements at Mach 6," AIAA, Vol. 54, No. 1, 2015, pp. 53-65. doi:10.2514/1.J054139.

[12] Redford, J. A., Sandham, N. D., and Roberts, G. T., "Compressibility Effects on Boundary-Layer Transition Induced by an Isolated Roughness Element," AIAA Journal, 2010.

[13] Schneider, S. P., "Effects of Roughness on Hypersonic Boundary-Layer Transition,” AIAA, Vol. 45, 2007, pp. $193-209$. doi:10.2514/1.29713. 
[14] Saric, W. S., Reed, H. L., and B.White, E., "Stability and transtion of three-dimensional boundary layers," Annu. Rev. Fluid Mech., Vol. 35, 2003, pp. 413-440. doi:10.1146/annurev.fluid.35.101101.161045.

[15] Saric, W. S., "Görtler vortices," Annual Review of Fluid Mechanics, Vol. 26, No. 1, 1994, pp. 379-409. doi:10.1146/annurev.fl. 26.010194.002115.

[16] Reshotko, E., "Role of Transient Growth in Roughness-Induced Transition," AIAA Journal, Vol. 42, No. 4, 2004 , pp. 766-770. doi:10.2514/1.9558.

[17] Citro, V., Giannetti, F., Luchini, P., and Auteri, F., "Global stability and sensitivity analysis of boundary-layer flows past a hemispherical roughness element," Physics of Fluids, Vol. 27, No. 8, 2015, p. 084110. doi:10.1063/1.4928533.

[18] Brynjell-Rahkola, M., Shahriari, N., Schlatter, P., Hanifi, A., and Henningson, D. S., "Stability and sensitivity of a cross-flowdominated Falkner-Skan-Cooke boundary layer with discrete surface roughness," Journal of Fluid Mechanics, Vol. 826, 2017, pp. 830-850.

[19] Loiseau, J.-C., Robinet, J.-C., Cherubini, S., and Leriche, E., "Investigation of the roughness-induced transition: global stability analyses and direct numerical simulations," Journal of Fluid Mechanics, Vol. 760, 2014, pp. 175-211.

[20] Carpenter, M. H., Nordstrom, J., and Gottlieb, D., "A Stable and Conservative Interface Treatment of Arbitrary Spatial Accuracy," Journal of Computational Physics, Vol. 365, No. 98, 1998, pp. 341-365.

[21] Jacobs, C. T., Jammy, S. P., and Sandham, N. D., "OpenSBLI: A framework for the automated derivation and parallel execution of finite difference solvers on a range of computer architectures," Journal of Computational Science, Vol. 18, 2017, pp. 12-23.

[22] Lusher, D. J., Jammy, S. P., and Sandham, N. D., "Shock-wave/boundary-layer interactions in the automatic source-code generation framework OpenSBLI," Computers \& Fluids, Vol. 173, 2018, pp. 17 - 21.

[23] Fu, L., Hu, X. Y., and Adams, N. A., "A family of high-order targeted ENO schemes for compressible-fluid simulations," Journal of Computational Physics, Vol. 305, 2016, pp. 333-359.

[24] Kim, K. H., Kim, C., and Rho, O.-H., "Methods for the accurate computations of hypersonic flows. I. AUSMPW+ scheme." Journal of Computational Physics, Vol. 174, No. 1, 2001, pp. 38-80.

[25] Lele, S. K., “Compressibility effects on turbulence,” Annual Review Fluid Mechanics, Vol. 26, No. 1, 1994, pp. $211-254$. doi:10.1146/annurev.fl.26.010194.001235.

[26] Visbal, M. R., and Gaitonde, D. V., "On the Use of Higher-Order Finite-Difference Schemes on Curvilinear and Deforming Meshes," Journal of Computational Physics, Vol. 181, 2002, pp. 155-185.

[27] White, F. M., Viscous Fluid Flow, $3^{\text {rd }}$ ed., Mc McGraw-Hill, 2006.

[28] De Tullio, N., and Sandham, N., "Influence of boundary-layer disturbances on the instability of a roughness wake in a high-speed boundary layer," Journal of Fluid Mechanics, Vol. 763, 2015, pp. 136-165. doi:10.1017/jfm.2014.663.

[29] Chaudhry, R., Subbareddy, P., Nompelisy, I., and Candler, G., "Direct Numerical Simulation of Roughness-Induced Transition in the VKI Mach 6 Tunnel," 2015. doi:10.2514/6.2015-0274.

[30] Iyer, P. S., Muppidi, S., and Mahesh, K., "Boundary layer transition in high-speed flows due to roughness," 50th AIAA Aerospace Sciences Meeting including the New Horizons Forum and Aerospace Exposition, 2012. doi:10.2514/6.2012-1106.

[31] De Tullio, N., Paredes, P., Sandham, N. D., and Theofilis, V., "Laminar-turbulent transition induced by a discrete roughness element in a supersonic boundary layer," Journal of Fluid Mechanics, Vol. 735, 2013, p. 613-646. doi:10.1017/jfm.2013.520. 